\title{
Ocorrência de Acanthocephala em Leontopithecus (Lesson, 1840), cativos: aspectos clínico- patológicos. Callitrichidae-Primates
}

\author{
[Acanthocephala infections in captives lion tamarins Leontopithecus (Lesson, 1840): clinical pathologic aspects. \\ Callitrichidae-Primates] \\ L. Pissinatti ${ }^{1,2}$, A. Pissinatti ${ }^{1,3,6 *}$, C.H.F. Burity ${ }^{4}$, D.G. Mattos $J r .^{5}$, R. Tortelly ${ }^{5}$ \\ ${ }^{1}$ Centro de Primatologia do Rio de Janeiro - FEEMA \\ Estrada do Paraíso, $\mathrm{s} / \mathrm{n}$ \\ 25940-000 - Guapimirim, RJ \\ ${ }^{2}$ Aluno de pós-graduação - FV-UFF - Niterói, RJ \\ ${ }^{3}$ Fundação Educacional Serra dos Órgãos - Teresópolis, RJ \\ ${ }^{4}$ Universidade do Rio de Janeiro - Rio de Janeiro, RJ \\ ${ }^{5}$ Faculdade de Veterinária - UFF - Niterói, RJ \\ ${ }^{6}$ Centro Universitário Plínio Leite - Niterói, RJ
}

\begin{abstract}
RESUMO
Estudaram-se achados de necropsia de 454 espécimens de Leontopithecus. Quatorze (3,1\%) apresentaram-se infectados por acantocéfalos intestinais identificados como Prosthenorchis elegans (Diesing, 1861). Não foram observadas diferenças na ocorrência do parasito quanto à espécie, origem cativos ou selvagens - e sexo. Clinicamente os animais apresentaram-se com o pelame eriçado, apatia, inapetência, dores abdominais e diarréia. Em dois animais parasitados (14,2\%), observou-se perfuração da parede intestinal. As lesões encontradas caracterizaram-se por uma grave enterite ulcerativa. A ocorrência desses parasitos reforça a necessidade do estabelecimento de protocolos sanitários rígidos no manejo das espécies de primatas do neotrópico.
\end{abstract}

Palavras-chave: mico-leão, Leontopithecus, acontocéfalo, patologia

\section{ABSTRACT}

This study assessed the autopsy findings of 454 Leontopithecus specimens, 14 of which (3.1\%) were infected by intestinal Acanthocephala identified as Prosthenorchis elegans (Diesing, 1861). No difference in the occurrence of the parasite was observed regarding the species, origin (captive or wild) and sex of the lion tamarins. Clinically, the animals had spiked coat, apathy, inappetence, abdominal pain and diarrhea. Two of the parasitized animals (14.2\%) had perforation of the intestinal wall. The lesions found were characterized as a severe ulcerative enteritis. The occurrence of such parasites highlights the need for establishing strict sanitary protocols for the management of the neotropical primate species.

Keywords: Lion tamarins, Leontopithecus, Acanthocephala, pathology

\section{INTRODUÇ̃̃O}

O território brasileiro possui grande diversidade de habitat, fato que favoreceu sua extraordinária riqueza primatológica, aliás a mais significativa dentre todas as unidades políticas mundiais (Coimbra-Filho, 1973; Mittermeier et al., 1994).

Os micos-leões (Leontopithecus) são as espécies mais corpulentas da família Callitrichidae, habitando remanescentes da Mata Atlântica

Recebido em 19 de junho de 2006

Aceito em 5 de novembro de 2007

*Autor para correspondência (corresponding author)

E-mail: pissinatticprj@terra.com.br 
Brasileira. Todas as quatro formas se encontram criticamente ameaçadas: Leontopithecus rosalia no estado do Rio de Janeiro, Leontopithecus chrysopygus no estado de São Paulo Leontopithecus caissara do extremo norte do estado do Paraná e sudeste de São Paulo e Leontopithecus chrysomelas, restrito a florestas no sudeste do estado da Bahia (Coimbra-Filho, 1976; Ballou et al., 1997).

Relativamente à sobrevivência desse gênero, Coimbra-Filho e Mittermeier (1973) realizaram vários estudos. Beck et al. (1991) ensaiaram reintroduções e programas de educação ambiental, principalmente com L. rosalia. Tais estudos, porém, são preliminares com $L$. caissara, que ainda não possui qualquer colônia ex situ (Ballou, 1989; Ballou et al., 1997).

Para o sucesso dos programas de propagação ex situ, torna-se indispensável a participação de veterinário especializado, com sólidos conhecimentos da anatomia, fisiologia e patologia desses táxons (Montali e Bush, 1999). Infelizmente permanecem escassos os trabalhos publicados sobre aspectos da morfologia e da patologia do gênero Leontopithecus, pois a literatura ainda é insuficiente para um melhor conhecimento desses símios (Pissinatti et al., 1993; Burity et al., 1997).

Os acantocéfalos pertencem ao ramo em que todos os representantes são parasitas obrigatórios. Apresentam uma tromba retrátil que no extremo anterior está provida de ganchos (Read, 1974). São pseudocelomados dióicos, com ausência de trato digestivo; alimentam-se por meio da parede cuticular, à semelhança dos cestóides (Travassos, 1917).

As espécies de acantocéfalos descritas na literatura como parasitas de primatas do Novo Mundo são apenas três: Prosthenorchis elegans, P. spirula e Moniliformis clarki. Os adultos de Prosthenorchis sp habitam o lúmem da porção terminal do íleo, do ceco e do cólon dos platirrinos, fixando-se firmemente à parede intestinal por meio da inserção profunda de suas probóscides espinhosas na submucosa e túnica muscular do intestino (Dunn, 1963; Nelson et al., 1966).

Os estudos de Brumpt e Urbain (1938) demonstraram que $P$. elegans e $P$. spirula têm uma variedade muito grande de hospedeiros, podendo também ser um provável parasito de humanos.

O presente trabalho tem como objetivo registrar a ocorrência de infecções causadas por Acantocephala em primatas brasileiros mantidos em cativeiro e em condições especiais de manejo.

\section{MATERIAL E MÉTODOS}

Os dados dos micos-leões utilizados neste estudo são provenientes do acervo do Museu do Centro de Primatologia do Rio de Janeiro (FEEMA) (Coimbra-Filho et al., 1986). Os primatas foram necropsiados e a carcaça e as vísceras foram fixadas por imersão em solução de formol tamponado a $10 \%(\mathrm{pH} 7,2)$. Foram examinados 454 protocolos de necropsia dentre as quatro espécies do táxon, de acordo com a Tab. 1.

Os fragmentos dos órgãos e tecidos de cada um dos animais positivos amostrados foram encaminhados ao laboratório de histopatologia e processado de acordo com as técnicas de rotina de inclusão em parafina, cortados com $5 \mu \mathrm{m}$ de espessura e corados em hematoxilina-eosina. Os diagnósticos histopatológicos foram feitos com o auxílio de microscópio óptico Olympus.

Os dados obtidos foram tabulados em planilha eletrônica (Excel - Microsoft ${ }^{\circledR}$ ), e analisados pelo teste não paramétrico do qui-quadrado. $\mathrm{O}$ teste foi empregado para avaliar as possíveis diferenças entre a incidência de acantocéfalos nas diferentes espécies de Leontopithecus. Em se tratando de diferenças entre proporções de afetados ou do sexo, foi utilizado um teste para comparar as diferenças entre proporções $-z$-test. O nível de significância adotado foi de $\alpha=0,05$ (Zar, 1984). Os animais do táxon L. caissara não foram analisados estatisticamente, pois somente três espécimes foram estudados e todos negativos para Acanthocephala.

\section{RESULTADOS}

Os acantocéfalos foram identificados como pertencentes à família Oligacanthorynchidae, espécie Prosthenorchis elegans (Diesing, 1861) Travassos, 1915. 
Ocorrência de Acanthocephala...

Tabela 1. Distribuição da amostra segundo a espécie de Leontopithecus por sexo, origem e faixa etária e número de animais infectados (Inf) por Prosthenorchis elegans

\begin{tabular}{|c|c|c|c|c|c|c|c|c|c|c|c|c|c|}
\hline \multirow{3}{*}{ Espécie } & \multirow{3}{*}{ Total } & \multicolumn{6}{|c|}{ Macho } & \multicolumn{6}{|c|}{ Fêmea } \\
\hline & & \multicolumn{3}{|c|}{ Silvestre } & \multicolumn{3}{|c|}{ Cativeiro } & \multicolumn{3}{|c|}{ Silvestre } & \multicolumn{3}{|c|}{ Cativeiro } \\
\hline & & Jovem & Adulto & Inf & Jovem & Adulto & Inf & Jovem & Adulto & Inf & Jovem & Adulto & Inf \\
\hline L. rosalia & 186 & 1 & 17 & 0 & 9 & 66 & $1(0,5 \%)$ & 0 & 26 & $1(0,5 \%)$ & 11 & 56 & $2(1 \%)$ \\
\hline L. chrysomelas & 122 & 1 & 17 & $1(0,8 \%)$ & 11 & 24 & 0 & 4 & 18 & $2(1,6 \%)$ & 17 & 30 & $1(0,8 \%)$ \\
\hline L. chrysopygus & 143 & 0 & 5 & 0 & 14 & 59 & $6(4,1 \%)$ & 0 & 7 & 0 & 8 & 50 & 0 \\
\hline L. caissara & 3 & 0 & 1 & 0 & 0 & 0 & 0 & 0 & 2 & 0 & 0 & 0 & 0 \\
\hline Total & 454 & 2 & 40 & $1(0,2 \%)$ & 34 & 149 & $7(1,5 \%)$ & 4 & 53 & $3(0,6 \%)$ & 36 & 136 & $3(0,6 \%)$ \\
\hline
\end{tabular}

As informações dos exames anatomopatológicos e clínicos contidos nos protocolos de cada um dos micos-leões do período compreendido entre 1979 e 2001, da colônia do CPRJ-FEEMA indicaram a presença de parasitos intestinais acantocéfalos - que foram identificados como Prosthenorchis elegans, segundo Machado Filho (1950). Dos 454 indivíduos examinados, 14 $(3,1 \%)$ apresentaram a parasitose que incluiam as espécies L. rosalia, L. chrysomelas e $L$. chrysopygus (Tab. 1). O número de espécimens de parasitos encontrados por animal variou de 2 a 32. (Fig. 1 e 2).

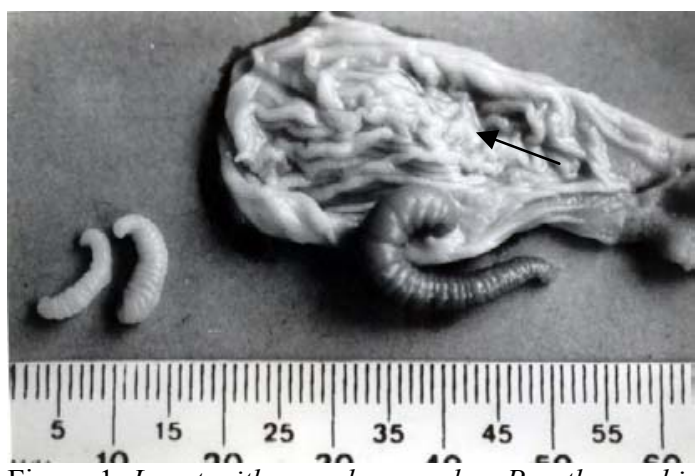

Figura 1. Leontopithecus chrysomelas. Prosthenorchis elegans fixado à camada muscular intestinal; seta indicando onde estava fixado outro parasito.

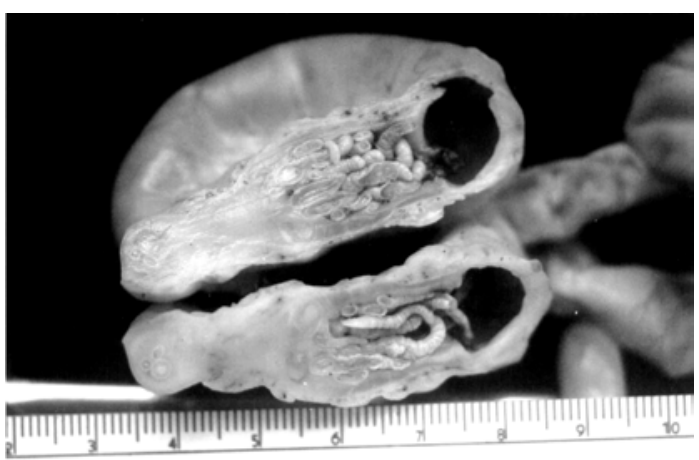

Figura 2. Leontopithecus rosalia. Vários exemplares de Prosthenorchis elegans obstruindo a válvula ileocecal. Notar o espessamento da parede intestinal.
A prevalência foi independente da espécie $\left(\chi^{2}=1,940 ;\right.$ G.L. $\left.=2 ; \mathrm{P}=0,379\right)$.

As comparações entre as proporções de afetados levaram em conta o caráter sexo - macho ou fêmea - e a origem - cativo ou de vida livre. Os valores obtidos de $z$ e o nível de significância para as espécies L. rosalia (sexo: $z=0,707$, $\mathrm{P}=0,480 ; \quad$ origem: $\quad z=0,707, \quad \mathrm{P}=0,480), \quad L$. chrysomelas (sexo: $z=0,566, \mathrm{P}=0,572$, origem: $z=0,638, \quad \mathrm{P}=0,523) \quad e \quad$ L.chrysopygus (sexo/origem: só machos e cativos na análise) não diferiram entre si.

O exame microscópico revelou na luz ou na parede dos intestinos múltiplos parasitos fixados (Fig. 3), que estavam associados a uma grave enterite ulcerativa, mostrando um rico infiltrado inflamatório misto difuso. Em torno dos helmintos fixados à camada muscular, observouse uma reação inflamatória crônica fibrosa acompanhada de discreto infiltrado leucocitário. Freqüentemente a serosa adjacente mostrava-se invadida por mononucleares.

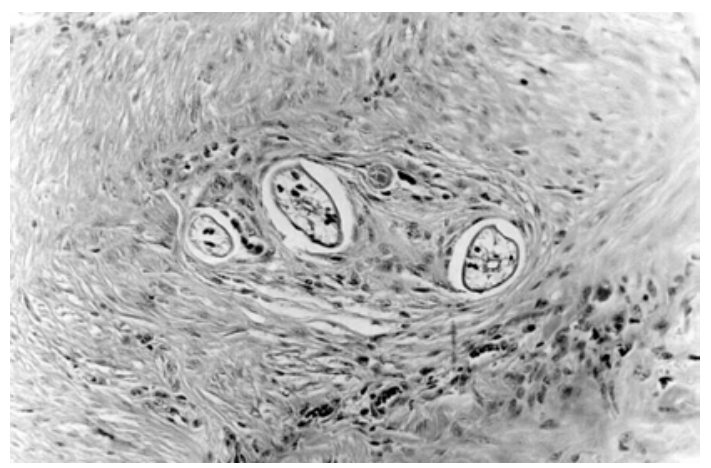

Figura 3. Leontopithecus rosalia. Camada muscular intestinal, fragmentos de parasitos em meio a tecido fibroso. HE 200X.

A perfuração nos intestinos foi observada macroscopicamente em $L$. rosalia e $L$. chrysomelas, representando $14,2 \%$ dos 14 casos estudados. 
As alterações clínicas dos animais parasitados dependeram da severidade da infecção. Os animais apresentaram, em geral, pelame eriçado, apatia, inapetência, dores abdominais, diarréia, emagrecimento progressivo seguido de debilidade física, associada a complicações secundárias, dor e morte.

\section{DISCUSSÃO}

No gênero Leontopithecus, apenas em L. rosalia foi observada a presença de Prosthenorchis elegans (Dunn, 1963; Stunkard, 1965). Para L. chrysomelas e L. chrysopygus, até a presente data, esta constitui a primeira citação na literatura.

A ocorrência de $P$. elegans no trato intestinal constitui um achado preocupante, devido à ineficácia dos medicamentos até agora empregados, mesmo quando associados às boas técnicas de manejo e, principalmente, por se tratarem de espécies primatas seriamente ameaçadas de extinção.

Os micos-leões encontravam-se parasitados quando vieram a óbito, sendo quatro $L$. rosalia, quatro $L$. chrysomelas e dois L. chrysopygus. A presença desse parasita foi determinante para a morte dos animais, como fora observado em Callithrix geoffroyi por Pereira Leite (2002), em animais do Brasil, e por Arrojo (2002) no Peru.

Os vários graus de diarréia, anorexia, debilidade e morte foram também observados em Nasua narica (Chandler, 1953) Callithrix (Takos e Thomas, 1958; Deinhardt et al. 1967), Saguinus (Nelson et al. 1966) e Saimiri (Arrojo, 2002).

Segundo Takos e Thomas (1958), Dunn (1963), Nelson et al. (1966) e King Jr. (1993), Prosthenorchis sp. é importante não apenas pela sua alta patogenicidade, mas também porque é hospedeiro intermediário da barata (Blatella germanica), que é encontrada nos criatórios de primatas e participa do ciclo dessa parasitose (Brumpt e Urbain, 1938; Graham, 1960).

Não existem muitos relatos de tratamentos medicamentosos eficazes contra estes parasitos, devendo-se dar toda ênfase ao controle dos hospedeiros intermediários, com o intuito de prevenir a ocorrência do parasitismo (Demidov et al., 1988; King Jr., 1993; Weber e Junge, 2000).
Em relação às perfurações intestinais, os registros na literatura são raros, nem sempre também estão relacionadas ao intenso parasitismo, porque hemorragias intestinais e até perfurações da parede podem ocorrer na presença de número muito baixo de parasitas no lúmen, enquanto, externamente, só se observam nódulos reacionais (Dunn,1963; Pereira Leite, 2002).

Vale ressaltar que os micos-leões de vida livre parasitados passaram por zoológicos, onde poderiam ter se infectado. O cativeiro é o locus onde há maior possibilidade de infecção de hospedeiros definitivos, devido à diversidade de espécies de primatas mantidas conjuntamente, associada ao manejo deficiente.

Em L. rosalia que vivia em habitat natural já foi diagnosticada P. elegans (Monteiro et al., 2003). A origem dessa parasitose pode estar relacionada à presença de espécies invasoras como Callithrix jacchus e $C$. penicillata ali introduzidas $\mathrm{e}$ resultantes do tráfico de animais que foram soltos de modo indiscriminado, sem triagem clínica apurada. Essa prática de controle é seguida no processo de repovoamento com os micos-leões dourados (Bush et al., 1993; Montali e Bush, 1999). Os resultados apresentados sugerem que esse parasitismo acomete principalmente os adultos de ambos os sexos, uma vez que não foi observado em animais impúberes.

Os achados desse parasitismo, bem como as lesões associadas são compatíveis com as observações de outros investigadores, reforçando a necessidade de se estabelecerem protocolos sanitários rígidos no manejo dos primatas da região neotropical. Levando-se em conta a possibilidade de epizootias, já ocorridas em vários países, são da maior importância o controle sanitário e a quarentena dos animais de zoológicos ou entidades com atividades semelhantes como os núcleos de criação.

\section{AGRADECIMENTOS}

Ao Dr. Arandas Rego da Fundação Instituto Oswaldo Cruz (FIOCRUZ), pela identificação dos acantocéfalos. A Fundação Carlos Chagas Filho de Amparo à Pesquisa do Estado do Rio de Janeiro (Proc. N $N^{\circ}$ E-26/171.185/2004). Ao Greater Los Angeles Zoo Association (GLAZA) e ao IBAMA, pela constante cooperação no programa de reprodução de primatas do neotrópico. 


\section{REFERÊNCIAS BIBLIOGRÁFICAS}

ARROJO, L. Parasitos de animales silvestres en cautiverio en Lima, Peru. Rev. Peru. Biol., v.9, p.118-120, 2002.

BALLOU, J.D. Emergence of the captive population of golden-headed lion tamarins Leontopithecus chrysomelas. Dodo J. Jersey Wildl. Preserv. Trust, v.26, p.70-77, 1989.

BALLOU, J.D.; LACY, R.C.; ELLIS, S. (compilers): Leontopithecus II: The second population and habitat viability assessment for Lion Tamarins (Leontopithecus). IUCN/SSC Conservation Breeding Specialist Group (CBSG): Apple Valley, Minnesota, 1997.

BECK, B.B.; KLEIMAN, D.G.; DIETZ, J. et al. Losses and reproduction in reintroduced golden lion tamarins Leontopithecus rosalia. Dodo J. Jersey Wildl. Preserv. Trusty., v.27, p.50-61, 1991.

BRUMPT, R.; URBAIN, A. Une curieuse épizootie vermineuse à acanthocéphales, devenue endèmique à la singerie du Muséum. Mesures prophylatiques eficaces prises pour en arrêter lês mèfaits. C.R. Acad. Sci. Paris, v.206, p.1927-1930, 1938.

BURITY， C.H.F.; MANDARIM-DE-LACERDA， C.A.; PISSINATTI, A. Craniometric Sexual Dimorphism in Leontopithecus Lesson, 1840 (Callitrichidae, Primates). Primates, v.38, p.101-108, 1997.

BUSH, M.; BECK, B.B.; MONTALI, R.J. Medica considerations of reeintroduction. In: FOWLER, M.E. Zoo and Wild Animal Medicine. Current Therapy. London: W.B. Saunders. 1993. v.3, p.24-26.

CHANDLER, A.C. An outbreak of Prosthenorchis (Acanthocephala) infection in primates in the Houston Zoological Garden, and a report of this parasite in Nasua narica in Mexico. J. Parasitol., v.39, p.226, 1953.

COIMBRA-FILHO, A.F. Os sagüis do gênero Leontopithecus Lesson, 1840. Callitrichidae - Primates. 1976. 72f. Dissertação (Mestrado) - Universidade Federal do Rio de Janeiro, Rio de Janeiro.

COIMBRA-FILHO, A.F.; MITTERMEIER, R.A. Distribution and ecology of the genus Leontopithecus in Brazil. Primates, v.14, p. 47-66, 1973.

COIMBRA-FILHO, A.F.; PISSINATTI, A.; SILVA, R.R. O acervo do Museu de Primatologia (CPRJ-FEEMA). In: MELLO, M.T. (Ed.). A Primatologia no Brasil, 2., Campinas, 1986. p.505-514.

DEINHARDT, J.B.; DEVINE, J.; PASSOVOY et al. Marmosets as laboratory animals: 1- care of marmosets in the laboratory pathology and outline of statistical evaluation of data. Lab. Anim. Care, v.17, p.191-214, 1967.

DEMIDOV, N.V.; KHRUSTALEV, A.V.; MOVCHAN, A.T. Usovershenstovanie metodov diagnostiki I terapii pri gel'mintozakh. Trudy Vsesoyuznogo Instit. Gel'mintologii im, v.29, p.41-46, 1988.

DUNN, F.L. Acanthocephalas and Cestodes of South American Monkeys and Marmosets. J. Parasitol., v.49, p.717-722, 1963.
GRAHAM, G.L. Parasitism in monkeys. Ann. N.Y. Acad. Sci., , v.85, p.842-860, 1960.

KING, JR., N.W. Prosthenorchiasis. In: JONE, T.C.; MOHR, U.; HUNT, R.D. (Eds.). Nonhuman primates II. Berlin: Springer-Verlag, 1993. p.65-68.

MACHADO FILHO, D.A. Revisão do gênero Prosthenorchis Travassos 1915 (Acanthocephala). Mem. Inst. Oswaldo Cruz, v.48, p.495-544. 1950.

MITTERMEIER, R.A.; KONSTANT, W.R.; MAST, R.B. Use of neotropical and Malgasy Primates Species in Biomedical Research. Am. J. Primatol., v.34, p.73-80. 1994.

MONTALI, R.J.; BUSH, M. Diseases of Callitrichidae. In: FOWLER, R. (Ed.). Zoo and wild animal medicine: Current Therapy 4.ed. Philadelphia: W.B. Saunders; 1999. p.369-376.

MONTEIRO, R.V.; JANSEN, A.M.; PINTO, R.M. Coprological helminth screening in Brazilian free ranging golden lion tamarins, Leontopithecus rosalia (L., 1766) (Primates, Callitrichidae). Rev. Bras. Biol., v.63, p.727-729, 2003.

NELSON, B.; COSGROVE, G.E.; GENGOZIAN, N. Diseases of an imported primate Tamarinus nigricollis. Lab. Anim. Care, v.16, p.255-275, 1966.

PEREIRA LEITE, M.C. Patologia comparada de Callithrix geoffroyi, Humboldt, 1812 (Callitrichidae - Primates) mantidos em cativeiro no Centro de Primatologia do Rio de Janeiro. 2002. 206f. Tese (Doutorado) - Faculdade de Medicina Veterinária e Zootecnia, Universidade de São Paulo, São Paulo.

PISSINATTI, A.; SILVA Jr., E.C.; COIMBRA-FILHO, A.F. et al. Sexual dimorphism in Leontopithecus (Lesson, 1840). Folia Primatol., v.58, p.204-209, 1993.

READ, C.P. Parasitismo animal. São Paulo: Polígono, 1974, $223 p$.

STUNKARD, H.W. New intermediate Hosts in the life cycle of Prosthenorchis elegans (Diesing, 1851), an Acanthocephalan parasite of primates. J. Parasitol., v.51, p.645-649, 1965.

TAKOS, M.J.; THOMAS, L.J. The pathology and pathogenesis of fatal infections due to an acantocephalid parasite of marmoset monkeys. Am. J. Trop. Med. Hyg., v.7. p.90-94, 1958.

TRAVASSOS, L. Contribuição para o conhecimento da fauna helmintológica brasileira. Revisão dos acantocéfalos brasileiros. Parte I. Fam. Gigantorhynchidae Hamann, 1892. Mem. Inst. Oswaldo Cruz, v.9, p.5-62, 1917.

WEBER, M.; JUNGE, R. Identification and treatment of Moniliformis clarki (acanthocephala) in cotton-topped tamarins (Saguinus oedipus). J. Zoo Wild. Med., v.31, p.503507, 2000.

ZAR, H. Biostatistical analysis. Prentice Hall: Englewood Cliffs, 1999. 